\title{
3D Structural Analysis and Classification of EmaA, a Collagen Binding Adhesin
}

\author{
Claire J. Brooks ${ }^{1}$, Keith P. Mintz ${ }^{2}$, Michael Radermacher ${ }^{1}$, and Teresa Ruiz ${ }^{1}$ \\ ${ }^{1}$ Department of Molecular Physiology \& Biophysics, University of Vermont, Burlington, VT \\ ${ }^{2}$ Department of Microbiology \& Molecular Genetics, University of Vermont, Burlington, VT
}

Periodontal diseases represent a group of inflammatory conditions that lead to the destruction of periodontal tissues and are the most common cause of tooth loss in American adults. Aggregatibacter actinomycetemcomitans is associated with both the chronic and localized aggressive forms of periodontal diseases, as well as with infective endocarditis and urinary track infections. The binding of this Gram-negative bacterium to the host extracellular matrix collagen is mediated by the surface adhesin EmaA (extracellular matrix protein adhesin A), a virulence determinant ubiquitous in all $A$. actinomycetemcomitans serotypes. EmaA is one of the largest members of the trimeric autotransporter family with a monomer of $202 \mathrm{kDa}$ in molecular mass. The C-terminal regions of three EmaA monomers create a pore for translocation of the three N-terminal passenger domains across the outer membrane. These domains form antennae-like structures that project more than $150 \mathrm{~nm}$ away from the bacterial surface. The $760 \mathrm{~N}$-terminal amino acids of the EmaA monomers comprise the functional domain of the adhesin (Fig. 1) and are localized at the distal end of the appendage, within the most distal $30 \mathrm{~nm}$ [1]. The functional domain is further divided into three subdomains: SI-SIII, with SII and SIII separated by a linker region of $3 \mathrm{~nm}$ in diameter comprising a three $\alpha$-helix coiled-coil. Moreover, EmaA glycosylation was shown to be essential for its structural stability and collagen-binding ability [2]. To date, a high resolution structure of EmaA is lacking and the predictions from structural modeling programs are not compatible with available structural data. Thus, it is necessary to achieve a more detailed map of the secondary/tertiary/quaternary structure of the functional domain of EmaA.

EmaA adhesins from a serotype $b$ strain were analyzed by electron tomography of whole-mount negatively-stained preparations of the bacteria (Fig 2.a) [3]. Tomographic single-axis tilt series over a $\pm 64^{\circ}$ angular range in $2^{\circ}$ intervals were acquired with a calibrated pixelsize at the specimen scale of $0.308 \mathrm{~nm}$. The tilt series were processed using IMOD to generate tomograms. EmaA adhesins were selected from the tomograms by marking two points on their axis. For each selected adhesin, a tilt series of subprojections was extracted and subvolumes were calculated with the adhesin's axis approximately parallel to the Y-axis using algorithms in EMIRA [4]. The adhesin subvolumes were rotationally aligned to a preliminary 3-fold symmetrized averaged reference [1] by projection-based 3D volume alignment [5]. The calculated rotation was applied to the subprojections and translational shifts of the subvolumes were calculated by real space cross correlation. The aligned EmaA subvolumes underwent principle component analysis with expectation maximization (PPCA-EM) [6]. Outliers (with sigma of 5) were identified and excluded from this stage of the analysis. The PPCA-EM data was clustered using Diday's method of moving centers and representative 3D group averages were calculated by Radon reconstruction methods and used for further multireference alignment. Iterative cycles of multireference alignment and classification were performed to reconstruct the refined 3D structure of the different groups.

We have collected and processed 110 tomograms from serotype $\mathrm{b}$ A. actinomycetemcomitans and more than 300 EmaA functional domains were extracted from the tomograms. A total of $10 \%$ of the data was discarded after a preliminary selection for quality and presence of either cellular debris or gold fiducials 
within the projections at high tilt angles. An additional 5\% was deselected after the preliminary alignment step. The remaining $85 \%$ of the data after alignment were processed with PPCA-EM and showed seven major groups or clusters, all of them straight. In contrast, the structures of the groups obtained from glycosylation deficient strains exhibited a high degree of curvature within the functional domain. The largest group for the wild-type strain, GI, contained 60 members and showed high structural similarity with group GV (23 members) (Fig 2.b). A preliminary calculation of the resolution indicates that the GV cluster is much more homogeneous than GI. The second largest cluster GII (45 members) shows a slight kink after subdomain SIII and cluster GIII has a well-defined SII and a weaker density in SIII with a clear kink after the neck sequence N3. Further refinements of the structure are currently in progress.

References:

[1] Yu, C., Mintz, K.P., Ruiz, T., J. Bacteriol. 191 (2009), p.6253-6261.

[2] Tang, G., Ruiz, T., Mintz, K.P., Infect. Immun. 80 (2012), p.2868-2877.

[3] Azari, F., Radermacher, M., Mintz, K.P., Ruiz, T., J. Struct. Biol. 177 (2011), p.439-446.

[4] Radermacher, M., Microscopy \& Microanalysis, 19 (2013), Supplement S2 p.762-763.

[5] Yu, L. et al. Journal of Structural Biology 182 (2013), p. 93-105

[6] Yu, L. et al. Journal of Structural Biology, 171 (2010), p.18-30

[7] This work was supported by NIH grant DE024554, (T.R. \& K.P.M) and GM078202 (M.R.). We would like to thank Dr. F. Azari for help with processing the tomography data.

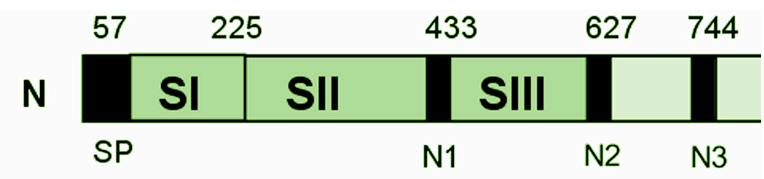

Figure 1. Sequence of the functional domain of EmaA showing the location of the SI-SIII subdomains and the N1-N3 neck sequences. The linker region corresponds to N1.

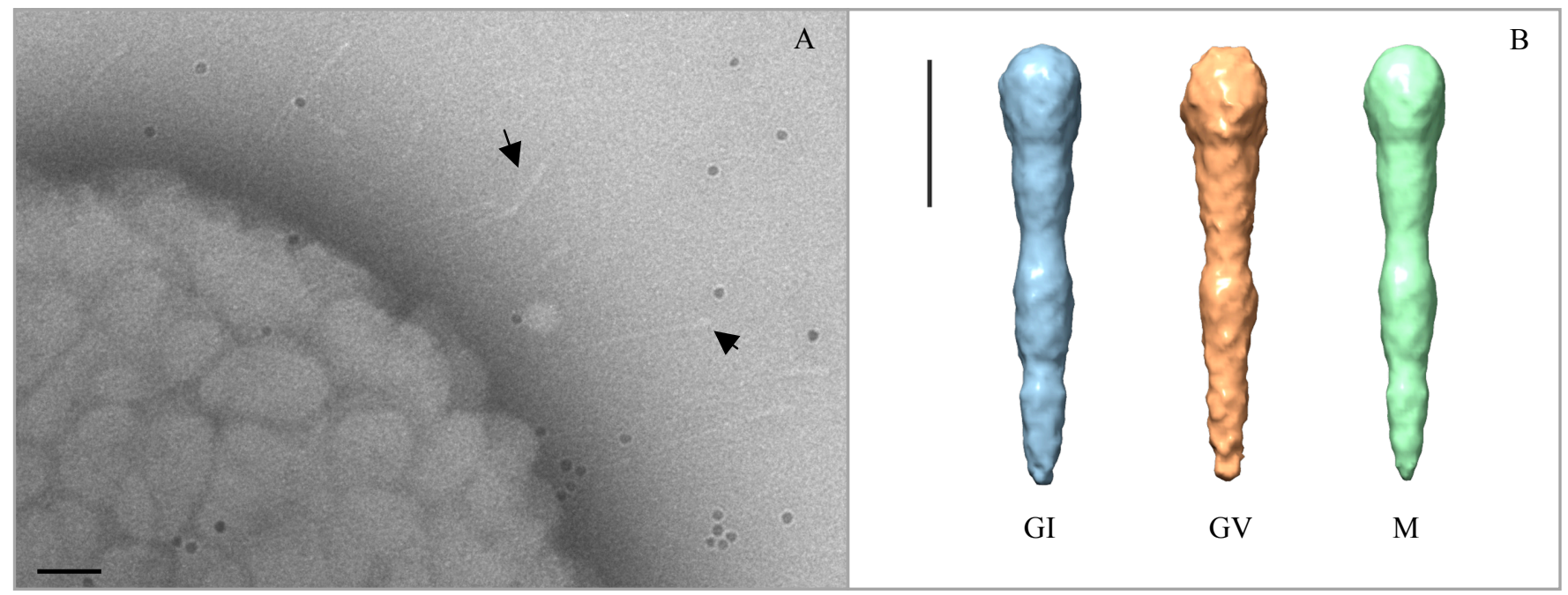

Figure 2. Structural analysis of the wild-type EmaA functional domain (A) Electron micrograph, $0^{\circ}$ projection. Bar: $50 \mathrm{~nm}$. Arrows point to representative EmaA adhesins. (B) Surface representations of the 3-fold symmetrized EmaA subgroups GI, GV and the two groups merged (M). Bar: $10 \mathrm{~nm}$. 